\title{
Assessment of Liver Microcirculation in Human Cirrhosis
}

\author{
P.-Michel Huet, Carl A. Goresky, Jean-Pierre Villeneuve, Denis Marleau, \\ and John O. LOUGH, The Clinical Research Center and Department of \\ Medicine of the Hôpital Saint-Luc, Université de Montréal, and the McGill \\ University Medical Clinic in the Montreal General Hospital, Departments of \\ Medicine and Pathology of the Montreal General Hospital and McGill \\ University, Montreal, Quebec, Canada
}

A B S T R A C T Alterations in the liver microcirculation were characterized by use of the multiple-indicator dilution technique in 25 cirrhotic patients undergoing hemodynamic evaluation of portal hypertension. Hepatic vein outflow dilution curves were obtained after portal vein or hepatic artery injections of a vascular reference substance (labeled erythrocytes) and of diffusible substances (labeled albumin and sucrose). In 23 of these patients (19 with alcoholic cirrhosis and 4 with postnecrotic cirrhosis), unimodal erythrocytes and albumin curves were obtained; the immediately accessible albumin space ranged from normal values (that were substantially larger than the erythrocyte space) to low values (that were little larger than the erythrocyte space). In parallel with this, the hepatic extraction of indocyanine green decreased and was correlated with the albumin space $(r=0.821$, $P<0.001$ ). The form of labeled sucrose curves showed progressive changes indicating limited diffusion into the interstitial space. In contrast, bimodal curves were found in two patients (with macronodular cirrhosis); a large proportion of all labels appeared simultaneously in the early part of the outflow curves. Model analysis of the unimodal data indicated that the spectrum of findings could best be explained by progressive development of a barrier to exchange by progressive capillarization of the microvascular bed, and the form of the bimodal data suggested that large vessel shunting was occurring. Both changes, in turn, will contribute to the reduced extraction of protein-bound materials in cirrhosis.

Dr. Huet is a Chercheur-Boursier of the Ministère des Affaires Sociales du Québec, Dr. Goresky is a Career Investigator of the Medical Research Council of Canada, and Dr. Villeneuve is a Scholar of the Canadian Liver Foundation. Address reprint requests to Dr. Huet, Clinical Research Center, Hôpital Saint-Luc, 1058 St. Denis Street, Montreal, Quebec H2X 3J4, Canada.

Received for publication 10 July 1981 and in revised form 28 June 1982.

\section{INTRODUCTION}

As cirrhosis of the liver evolves, portal hypertension appears and, concomitantly, disorders are found in the ability of the liver to both extract materials from the blood and synthesize biologically important substances. Initial attempts to examine events at the level of liver circulation have centered around the measurement of liver blood flow. Usually, the extraction of an organic anionic substance, such as indocyanine green (ICG), ${ }^{1}$ is measured across the liver and from this and the infusion rate of the dye, the blood flow is estimated. Although the extraction of organic anions across the liver is high in normal livers, it is found to decrease in cirrhotic livers, sometimes to very low levels. This decreased extraction by the hepatocytes is not usually associated with changes in flow but correlates, rather, with a decrease in the Kupffer cell uptake of labeled albumin microaggregates (1). It has therefore been hypothesized that these findings in cirrhotic liver could be explained by changes at the microcirculatory level $(1,2)$ such as capillarization (3) or intrahepatic shunts (4).

We examined these possibilities by using the multiple-indicator dilution technique (5) in a group of cirrhotic patients who were undergoing hemodynamic assessment of portal hypertension. We characterized at the same time in these patients the hepatic vein outflow patterns resulting from portal vein or hepatic artery injection of labeled erythrocytes (RBC) and labeled plasma-dissolved substances. In the normal liver, plasma proteins such as albumin gain access to an extravascular space (the space of Disse) through fenestrae in and between the endothelial cells; this produces a major delay in, and decrease in magnitude of, the labeled albumin curve with respect to that of labeled

\footnotetext{
${ }^{1}$ Abbreviations used in this paper: ALB, albumin; DIF, diffusible substance; ICG, indocyanine green; $P$, peak; RBC, erythrocyte; SUC, sucrose; $t$, transit time; $t_{0}$, zero time.
} 
RBC (restricted to the vascular space), which can be analyzed using a flow-limited model (5). By contrast, in organs with a tight capillary circulation, where no significant albumin exchange occurs during the time of a single passage, the outflow dilution curve for labeled albumin is delayed only slightly in relation to the corresponding labeled $\mathrm{RBC}$ curve, by virtue of a minor intravascular separation process (6).

In the cirrhotic liver, labeled albumin and sucrose dilution patterns were found that ranged from that expected for a more or less normal liver to configurations approaching those characteristic of a tight capillary circulation. These changes were correlated with a progressive decrease of the extraction of ICG by the hepatocytes and provide evidence indicating that abnormalities of liver microcirculation play an important role in the pathophysiology of liver dysfunction in cirrhotic patients.

\section{METHODS}

Multiple-indicator dilution studies were carried out in 25 cirrhotic patients ( 23 males and 2 females) admitted to SaintLuc Hospital (Montreal, Quebec, Canada), who were undergoing a hemodynamic evaluation of portal hypertension. 19 patients had micronodular cirrhosis, which is thought to result from chronic alcoholism, and six patients had postnecrotic cirrhosis. Liver biopsy showed no evidence for active alcoholic hepatitis and neither jaundice nor ascites were present at the time of the study. Laboratory data in the patients studied were as follows: the serum albumin ranged from 2.0 to $4.5 \mathrm{~g} / 100 \mathrm{ml}(3.06 \pm 0.60$, mean $\pm \mathrm{SD})$; the increment in prothrombin time above control time ranged from 0 to $4.5 \mathrm{~s}(1.60 \pm 1.02)$; and the total bilirubin content ranged from 0.35 to $2.00 \mathrm{mg} / 100 \mathrm{ml}(1.20 \pm 0.47)$. The study had been approved by the local ethics committee and written informed consent was obtained from each patient. In 12 patients, right hepatic vein catheterization was performed $2 \mathrm{~d}$ after portal vein catheterization under fluoroscopic visualization (1); the tip of the portal catheter was positioned in the main portal vein, about $3 \mathrm{~cm}$ proximal to its bifurcation. In 13 patients, right hepatic vein catheterization was performed combined with a catheterization of the common hepatic artery by the Seldinger technique (7). Whenever possible, the catheter was advanced beyond the take-off of the gastroduodenal artery under fluoroscopic visualization, using a minimal amount of radiopaque material.

With the catheters in place, an indicator dilution study was carried out by injecting blood containing tracer materials into the portal vein or hepatic artery and collecting serial samples from the hepatic vein. The injection mixture contained ${ }^{51} \mathrm{Cr}$-labeled autologous $\mathrm{RBC}(2 \mu \mathrm{Ci})$ and either ${ }^{125}$-labeled $(2 \mu \mathrm{Ci})$ or ${ }^{99 \mathrm{~m}} \mathrm{Tc}$-labeled $(2 \mu \mathrm{Ci})$ albumin, in all instances, and ${ }^{3} \mathrm{H}$ - or ${ }^{14} \mathrm{C}$-labeled sucrose $(2 \mu \mathrm{Ci})$, in 11 instances. The injection mixture was placed in a $2.0-\mathrm{ml}$ flowthrough cuvette and, at a prearranged signal, was rapidly flushed (less than $1 \mathrm{~s}$ ) into the portal vein or hepatic artery with $10 \mathrm{ml}$ of heparinized blood or saline. Serial heparinized blood samples were collected from the hepatic vein catheter at rates of one sample per 1 or $2 \mathrm{~s}$, with a peristaltic pump set to a fixed flow (the rates varied from 45 to $60 \mathrm{ml} / \mathrm{min}$ ). The collecting system transit time was calculated from the sampling rate and the volume of the collecting system.
Standards were prepared from the injection mixture by the addition, in serial dilution, of blood obtained from the outflow catheter before the experimental run. An aliquot of sample or standard was diluted with saline, and assayed in an automatic dual channel well-type scintillation-crystal spectrometer for gamma rays of the appropriate energy. Pure activity standards were used to calibrate crossover corrections and the activity in each sample due to each labeled species ascertained. Where labeled sucrose was used, samples were centrifuged, and aliquots of the supernatant were diluted with saline, pipetted into scintillation fluid, and assayed separately in an automated liquid scintillation counter for the beta activity emitted by the labeled tritium or carbon in the sample. In all sucrose studies, ${ }^{99 \mathrm{~m}} \mathrm{Tc}$ albumin was used and a period of more than 10 half-lives was allowed to elapse before assay of the samples. Crossover standards indicated that no residual activity was present at this time.

The primary data to be dealt with in the indicator dilution studies are the outflow curves in the hepatic venous stream. To provide a basis for comparison between the tracers, the outflow activity was divided by the total amount injected (5), yielding a pattern expressed in terms of outflow fraction per milliliter of blood (i.e., a reciprocal volume). The outflow dilution curves were corrected for recirculation by linear extrapolation of the downslope to infinity on a semilogarithmic plot, and the areas under time outflow fraction per milliliter curves were calculated. The outflow recoveries of various labels were calculated as the ratio of their areas to that of labeled RBC (5). The mean transit times (transit time $[t]$ ) were measured by the procedure described by Meier and Zierler (8) and corrected by subtraction for those of outflow collecting system transit times.

\section{Calculations}

Description of the model. Goresky (5) has shown previously in the normal dog liver that with a flow-limited linear two-compartment model, the extravascular volumes of distribution of diffusible substances (DIF) such as labeled albumin and sucrose can be estimated from the displacement of their hepatic vein outflow curve relative to that of the vascular reference substance (RBC).

The ratio of the extravascular distribution volume accessible to the DIF over the vascular distribution volume $\left(\gamma_{\mathrm{DIF}}\right)$ can be obtained from $\left(1+\gamma_{D I F}\right)$ values computed as the ratio of the peak RBC outflow fraction per milliliter to the peak DIF outflow fraction per milliliter (5). Testing this flow-limited model, the form of the RBC curve beyond the time spent by labels in nonexchanging large vessels (zero time $\left.\left[t_{0}\right]\right)$, could be reproduced from the albumin and sucrose curves by decreasing the transit time for each point of the DIF curve by the factor $\left(1+\gamma_{D I F}\right)$ and because the areas under normalized outflow curves were the same, by increasing its magnitude by the factor $1 /\left(1+\gamma_{\text {DIF }}\right)(5)$. These findings strongly support the hypothesis that the flow-limited model adequately describes the diff usion of labeled albumin and sucrose in the extravascular space in the normal dog liver. At the present time, no satisfactory alternate way of accounting for the behavior of the diffusible substances in the normal liver has been proposed.

In the normal dog liver circulation, with the flow-limited model, the volume of distribution of labeled RBC, albumin, and sucrose was found to increase in that sequence; the relative space accessible to albumin was much larger than the RBC space (5). This contrasted with the behavior of labeled albumin in organs with tight capillary circulation, where its curve is only slightly delayed in relation to that of labeled 
RBC by virtue of minor intravascular separation probably due to interlaminar diffusion (9).

Application of the model. For each set of outflow dilution curves, the following data were calculated using the flowlimited distribution model. $\left(t_{0}\right)$, which corresponds to the time spent by labels in the large vessels, was calculated according to the formula $(5), t_{0}=\left[t_{\mathrm{PRBC}}\left(1+\gamma_{\mathrm{DIF}}\right)-t_{\mathrm{PDIF}}\right] /$ $\gamma_{\mathrm{DIF}}$, where $t_{\mathrm{PRBC}}$ is the transit time at the peak of the labeled RBC dilution curve, and $t_{\mathrm{P} \text { DIF }}$ is the transit time at the peak of the labeled DIF dilution curve; $\left(1+\gamma_{D I F}\right)$ was calculated as the ratio of the peak RBC outflow fraction per milliliter to the peak DIF outflow fraction per milliliter.

If events in the cirrhotic liver are similar to those in the normal dog liver, the DIF curves, beyond $t_{0}$, can be transformed, diminishing its time span and increasing its magnitude by the space ratio until it superimposes on the labeled RBC curves. We fitted a continuous function that could be interpolated to the data points and used the least-squares technique to find those values of $\gamma_{\text {DIF }}$ and $t_{0}$ that provided optimal superimposition of the transformed DIF curve on the reference curve (10).

Flow values were not calculated from the dilution studies because, as we have previously reported (7), hepatic blood flow cannot be accurately estimated from dilution curves after portal vein or hepatic artery injections in cirrhotic patients.

\section{Indocyanine green}

ICG studies were carried out in all but two of the patients. During a continuous infusion of dye $(0.5 \mathrm{mg} / \mathrm{min})$, samples were obtained from portal vein or hepatic artery and one hepatic vein (11). The hepatic extraction was measured and, from the rate of infusion and hepatic extraction, we calculated the hepatic blood flow, assuming that dye was being removed only by the liver (11). The portohepatic gradient was determined by measuring the difference between either free portal vein or wedged hepatic vein pressure and free hepatic vein pressure (7).

\section{Ultrastructural examinations}

In four of the patients studied with the protocols described above (and in an additional four patients with micronodular cirrhosis) an open wedge biopsy obtained at surgery was prepared for light and electron microscopy. The tissue was fixed in phosphate-buffered $2 \%$ glutaraldehyde, postfixed in osmium tetroxide, dehydrated through alcohol, and embedded in Epon 812. Regenerative and normal trabecular areas of parenchymal tissue were selected by phase-contrast light microscopy. Thin sections of these areas were stained with lead citrate and uranyl acetate for electron microscopy and 35- $\mathrm{mm}$ pictures were taken with a Philips 201 electron microscope (Philips Electronic Instruments, Mount Vernon, NY).

\section{RESULTS}

All patients were in a stable hemodynamic condition at the time of the study. The results are presented in Table I. In 23 patients, unimodal labeled RBC and albumin curves, with smooth monotonically decreasing downslopes, were obtained. In two patients (Nos. 24 and 25), the labeled RBC and albumin curves dis- played characteristics that lead one to infer that the labeled RBC and albumin pathways were bimodal. These two studies will be discussed later.

\section{Studies characterized by unimodal outflow dilution curves}

Labeled RBC and albumin studies. In the $23 \mathrm{pa}$ tients (19 with alcoholic cirrhosis and 4 with postnecrotic cirrhosis) with unimodal outflow dilution curves, the labeled albumin curve was displaced relative to that of labeled RBC as illustrated in Fig. 1. After an initial delay the outflow fraction per milliliter curve for labeled RBC rises to an early and high peak and then decays monotonically, until the slope is interrupted by recirculation of activity; it is thereafter extrapolated. The values for labeled albumin are lower on the upslope, the peak is lower and later, and the downslope decays more slowly. Although the changes are of the same kind in each panel, the relative magnitude of the differences between the labeled RBC and labeled albumin curves is large in the left-hand panel (patient 3) and much smaller in the right-hand panel (patient 17).
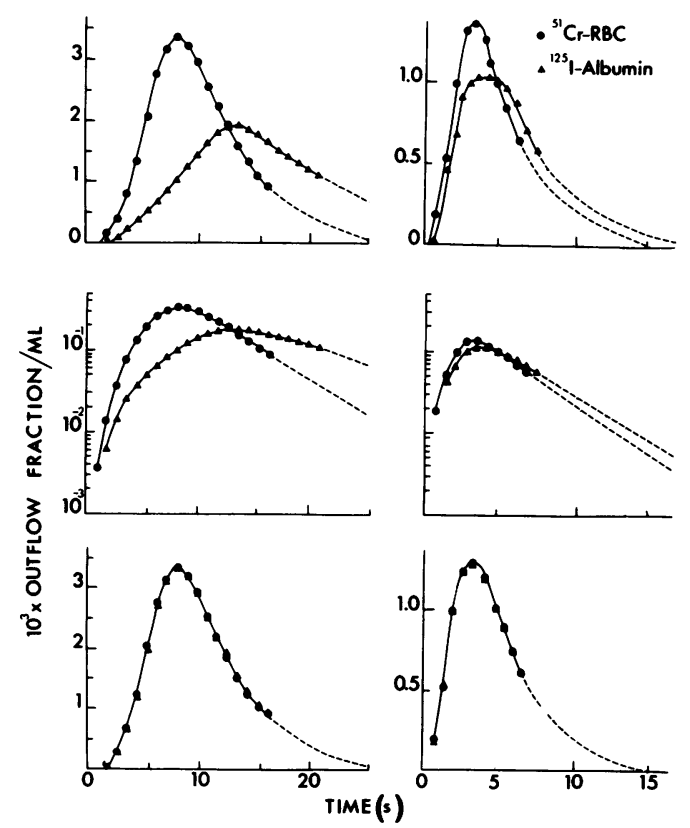

FIGURE 1 Labeled RBC and albumin outflow fraction per milliliter curves obtained in patients 3 (left-hand panel) and 17 (right-hand panel). The ordinate scale in the top panel is linear; in the middle panel, logarithmic; and in the bottom panel, linear. The bottom panel shows in each case the superimposition of the best-fit transformed, labeled albumin curve on the labeled RBC curve; the $\left(1+\gamma_{\mathrm{ALB}}\right)$ values were 1.83 and 1.15 , respectively; the coefficients of variation of the fit were 0.030 and 0.043 , respectively. 
TABLE I

ICG, Portohepatic Gradient, and Labeled RBC and Albumin Indicator Dilution Data

\begin{tabular}{|c|c|c|c|c|c|c|c|}
\hline No. & Diagnosis & $\begin{array}{c}\text { ICG } \\
\text { extraction }\end{array}$ & $\begin{array}{c}\text { Portohepatic } \\
\text { gradient }\end{array}$ & $\bar{t}_{\mathrm{RBC}} \cdot$ & $\bar{t}_{\mathrm{ALB}}{ }^{\circ}$ & $1+\gamma_{\text {ALBB }} t$ & $\begin{array}{l}\text { Liver blood } \\
\text { flow }\end{array}$ \\
\hline & & $(\%)$ & $(\mathrm{mm} \mathrm{Hg})$ & $(s)$ & $(s)$ & & $m l \min ^{-1} \mathrm{~kg}^{-1}$ \\
\hline 1 & AC & 36.9 & 12.5 & 9.72 & 15.25 & 1.80 & 30.0 \\
\hline 2 & $\mathrm{AC}$ & - & 6.5 & 10.35 & 16.42 & 1.87 & - \\
\hline 3 & AC & 36.6 & 9.0 & 15.21 & 24.24 & 1.83 & 29.0 \\
\hline 4 & AC & 32.2 & 15.0 & 8.32 & 12.94 & 1.91 & 19.2 \\
\hline 5 & $\mathrm{AC}$ & - & 9.5 & 10.82 & 14.29 & 1.59 & - \\
\hline 6 & $\mathrm{AC}$ & 9.1 & 9.0 & 12.92 & 16.39 & 1.40 & 50.1 \\
\hline 7 & PNC & 36.6 & 7.0 & 20.70 & 30.91 & 1.82 & 17.1 \\
\hline 8 & $\mathrm{AC}$ & 4.7 & 22.0 & 10.01 & 11.26 & 1.21 & 45.8 \\
\hline 9 & PNC & 44.1 & 21.0 & 15.46 & 21.91 & 1.94 & 10.0 \\
\hline $10 a$ & AC & 12.6 & 22.0 & 10.03 & 10.66 & 1.09 & 33.7 \\
\hline $10 \mathrm{~b}$ & - & 12.6 & 22.0 & 9.13 & 10.48 & 1.19 & 33.7 \\
\hline 11 & PNC & 30.7 & 24.5 & 11.39 & 15.63 & 1.61 & 32.9 \\
\hline 12 & AC & 5.9 & 26.5 & 8.20 & 9.58 & 1.27 & 31.7 \\
\hline 13 & $\mathrm{AC}$ & 17.7 & 18.0 & 9.49 & 14.78 & 1.62 & 12.0 \\
\hline $14 a$ & $\mathrm{AC}$ & 16.8 & 16.0 & 8.64 & 13.48 & 1.52 & 38.6 \\
\hline $14 \mathrm{~b}$ & - & 16.8 & 16.0 & 8.68 & 12.41 & 1.46 & 38.6 \\
\hline 15 & $\mathrm{AC}$ & 16.2 & 17.0 & 6.35 & 7.51 & 1.17 & 21.3 \\
\hline 16 & $\mathrm{AC}$ & 9.8 & 17.0 & 10.91 & 13.72 & 1.29 & 36.0 \\
\hline 17 & $\mathrm{AC}$ & 11.9 & 22.0 & 16.84 & 19.14 & 1.15 & 70.7 \\
\hline 18 & $\mathrm{AC}$ & 14.4 & 21.0 & 13.74 & 17.24 & 1.27 & 21.4 \\
\hline 19 & $\mathrm{AC}$ & 41.0 & 11.0 & 10.03 & 16.76 & 1.61 & 9.7 \\
\hline 20 & $\mathrm{AC}$ & 3.6 & 21.0 & 11.22 & 15.79 & 1.36 & 60.0 \\
\hline 21 & PNC & 47.6 & 22.0 & 10.33 & 16.33 & 1.70 & 7.6 \\
\hline 22 & AC & 60.3 & 10.0 & 17.25 & 27.31 & 1.80 & 13.4 \\
\hline 23 & $\mathrm{AC}$ & 11.6 & 22.0 & 11.24 & 15.29 & 1.45 & 15.4 \\
\hline 24 & PNC & 55.4 & 8.0 & Bimodal & Bimodal & - & 33.3 \\
\hline 25 & PNC & 25.8 & 19.0 & Bimodal & Bimodal & - & 20.2 \\
\hline
\end{tabular}

- The transit time is corrected for input and outflow delays.

$\downarrow$ The value tabulated is that resulting from a least-squares fit of the adjusted labeled albumin curve to the labeled RBC curve.

AC, alcoholic cirrhosis; PNC, postnecrotic cirrhosis.

In the 23 patients, the outflow recovery of labeled albumin was $0.98 \pm 0.05$ times that of labeled RBC, indicating that no labeled albumin was removed during a single passage through the cirrhotic liver.

The $\left(1+\gamma_{\text {ALB }}\right.$ [albumin, ALB] $)$ values cover a wide range (Table $\mathrm{I}$ ). Whereas in the normal dog the $\left(1+\gamma_{\mathrm{ALB}}\right)$ value is of the order of 1.5 to 1.9 , this value ranges from a maximum of 1.91 to a minimum of 1.09 in the cirrhotic liver. In two patients (Nos. 10 and 14), repeated dilution studies were carried out and the two successive $\left(1+\gamma_{\mathrm{ALB}}\right)$ values were nearly identical (Table I). The calculated $t_{0}$ was quite close to the point of appearance of labeled RBC, as occurred in the normal dog liver (5), and the average coefficient of variation of the fit when the transformed labeled albumin curve was fitted to the labeled RBC curve was 0.067 . The transformed labeled albumin curve did not differ from the labeled RBC curve systematically. The trans- formation was symmetrical, in the sense that when it was carried out in the opposite direction, to make the labeled RBC curve superimpose on the labeled albumin curve, the average relative degree of error (the coefficient of variation) was the same.

According to the flow-limited model, the delay in the transit time of labeled albumin relative to that of labeled RBC should be, for the 23 patients with unimodal curves, closely related to $\gamma_{\mathrm{ALB}}$. Fig. 2 illustrates the variation of $\gamma_{\mathrm{ALB}}$ with this, expressed as the excess albumin transit time ratio, $\left(t_{\mathrm{ALB}}-t_{\mathrm{RBC}}\right) / t_{\mathrm{RBC}}$; the variation is as expected. The data indicate that the displacement of the labeled albumin curve with respect to the labeled RBC curve is compatible with a flowlimited kind of behavior.

The data also clearly emphasize that a wide range of outflow dilution patterns can be obtained in cirrhotic livers: at one extreme, a pattern similar to that 


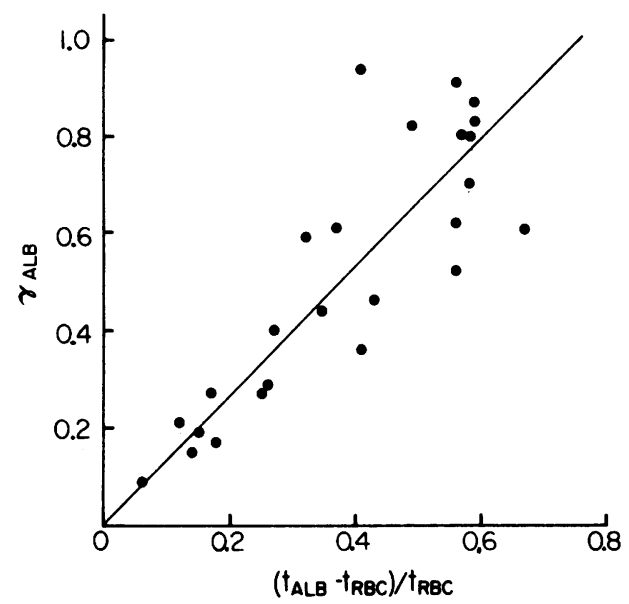

FIgURE 2 Plot of $\gamma_{\text {ALB }}$ vs. the excess albumin transit time ratio $\left(t_{\mathrm{ALB}}-t_{\mathrm{RBC}}\right) / t_{\mathrm{RBC}}$ in 23 patients. The solid line is the mean squares regression line through the data (12). The correlation coefficient for the data is $0.967(n=25, P<0.001)$.

expected in the normal liver was found, with a large separation between the labeled $\mathrm{RBC}$ and albumin curves, and a large $\gamma_{\mathrm{ALB}}$ value; and, at the other end, the labeled albumin curve approached that for labeled $\mathrm{RBC}$, and the $\gamma_{\mathrm{ALB}}$ estimate became so small that its value suggested that the vasculature had so changed that it had become almost impermeable to albumin over the time of a single transit. Benhamou et al. (13) previously suggested this hypothesis to explain low $\gamma_{\mathrm{ALB}}$ values in two of four cirrhotic patients.

Highly significant correlations were found between $\gamma_{\mathrm{ALB}}$ and the ICG extraction $(r=0.821, P<0.001)$ (Fig. 3) and between the excess albumin transit time ratio and the ICG extraction $(r=0.791, P<0.001)$. As the extraction of ICG decreases, both the $\gamma_{\mathrm{ALB}}$ and excess albumin transit time ratio are found to diminish. On the other hand, the relation between $\gamma_{\mathrm{ALB}}$ and the portohepatic gradient is less close with a large scatter in the data $(r=-0.551, P<0.01)$.

Labeled sucrose studies. In 9 of these 23 patients, labeled sucrose curves were also obtained. Fig. 4 (lefthand panel) illustrates the set of dilution curves obtained in patient 22. This patient had the least advanced cirrhosis in the group (he had a portohepatic gradient of $10 \mathrm{~mm} \mathrm{Hg}$ and an ICG extraction of $60.3 \%$ ) and one would therefore expect that the pattern of outflow profiles obtained might be close to that expected in a normal subject. The outflow patterns of the labeled albumin and sucrose were displaced relative to that of the RBC curve, showing a lower peak concentration and longer transit time, and the displacement was larger for sucrose than for albumin. The pattern resembled that found in the normal dog liver (5). In six other patients (No. 16, 18, 19, 20, 21,

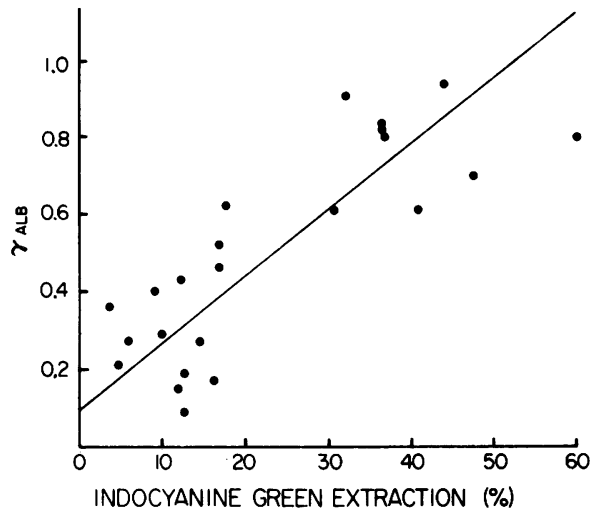

FIGURE 3 Correlation between $\gamma_{\mathrm{ALB}}$ and the ICG extraction. The solid line is the mean squares regression line through the data. The coefficient of correlation was $0.821(n=23$, $P<0.001$ ).

and 23), labeled sucrose curves exhibited displacements similar in kind; however, the delay in peak transit time was less than expected and the peak concentration was lower in magnitude than expected. A typical example is shown in the right-hand panel of Fig. 4 (patient 18). In two additional patients in whom the $\left(1+\gamma_{\mathrm{ALB}}\right)$ values were relatively low (patients 10 and 17), the sucrose curves exhibited a different and distinctive form; in each case the upslope was slightly delayed in relation to the labeled RBC curve, the labeled sucrose curve rose to a peak slightly lower and slightly ahead of that of the labeled albumin in time, and the downslope exhibited a biexponential character, the slower component of the downslope of the curve crossing that for labeled albumin. Fig. 5 (patient 10) provides a graphic illustration of this pattern.

The labeled sucrose curves did not, in general, have the kind of form expected for a material undergoing

TABLE II

Labeled RBC, Albumin, and Sucrose Indicator Dilution Data in the Nine Patients with Unimodal RBC and Albumin Curves

\begin{tabular}{lrrrrr}
\hline & & & & \\
No. & $t_{\mathrm{p} \mathrm{RAC}}$ & $t_{\mathrm{p} \mathrm{ALB}}$ & $t_{\mathrm{p} \mathrm{SUC}}{ }^{*}$ & $1+\gamma_{\text {ALB }}$ & $\begin{array}{c}\text { Apparent } \\
1+\gamma_{\mathrm{PSUC}}\end{array}$ \\
\hline & & $(s)$ & & & \\
$10 \mathrm{a}$ & 8.00 & 9.00 & 8.00 & 1.09 & 1.25 \\
16 & 9.12 & 11.12 & 13.12 & 1.29 & 5.87 \\
17 & 13.12 & 15.12 & 13.12 & 1.15 & 2.78 \\
18 & 9.12 & 11.12 & 16.12 & 1.27 & 5.20 \\
19 & 6.32 & 9.32 & 12.32 & 1.61 & 4.17 \\
20 & 8.12 & 11.12 & 15.12 & 1.36 & 5.65 \\
21 & 7.33 & 11.33 & 18.33 & 1.70 & 4.41 \\
22 & 12.11 & 18.11 & 28.11 & 1.80 & 3.48 \\
23 & 9.59 & 13.59 & 17.59 & 1.45 & 5.63 \\
\hline
\end{tabular}

- The peak transit time $\left(t_{\mathrm{p}}\right)$ is corrected for input and outflow delays. 

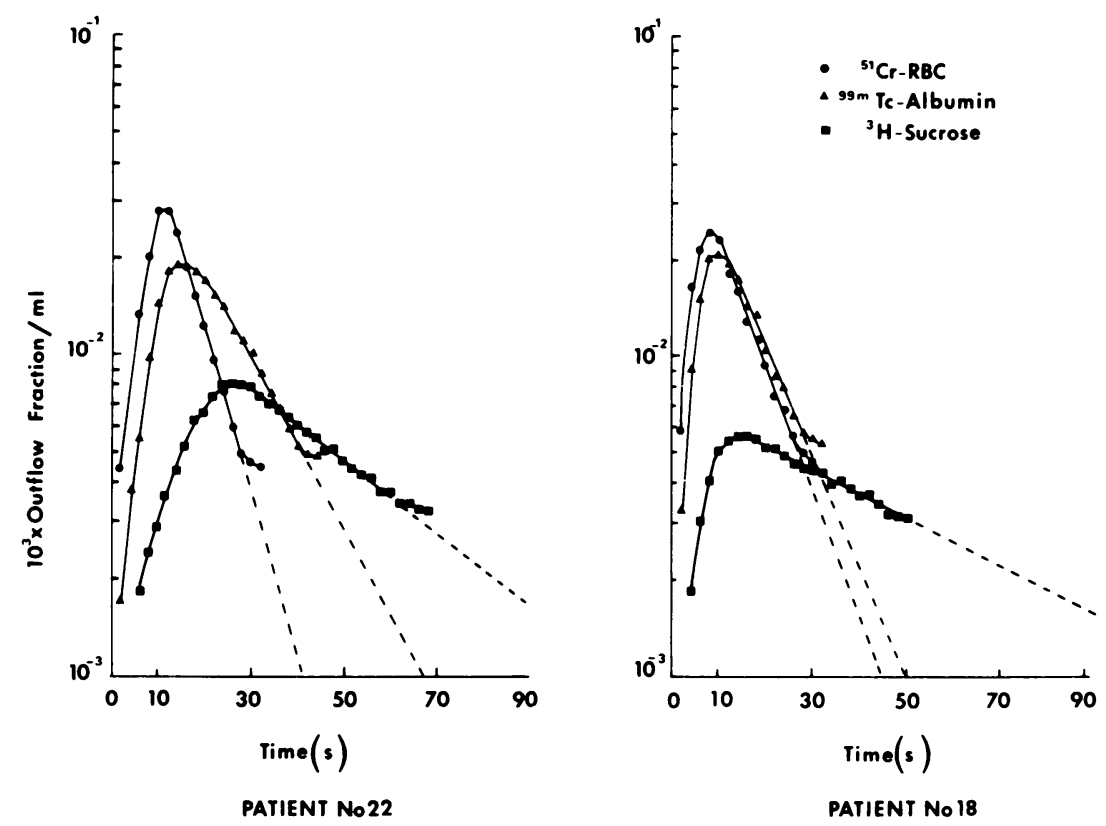

Figure 4 Labeled RBC, albumin, and sucrose outflow fraction per milliliter curves obtained in patients 22 (left-hand panel) and 18 (right-hand panel).

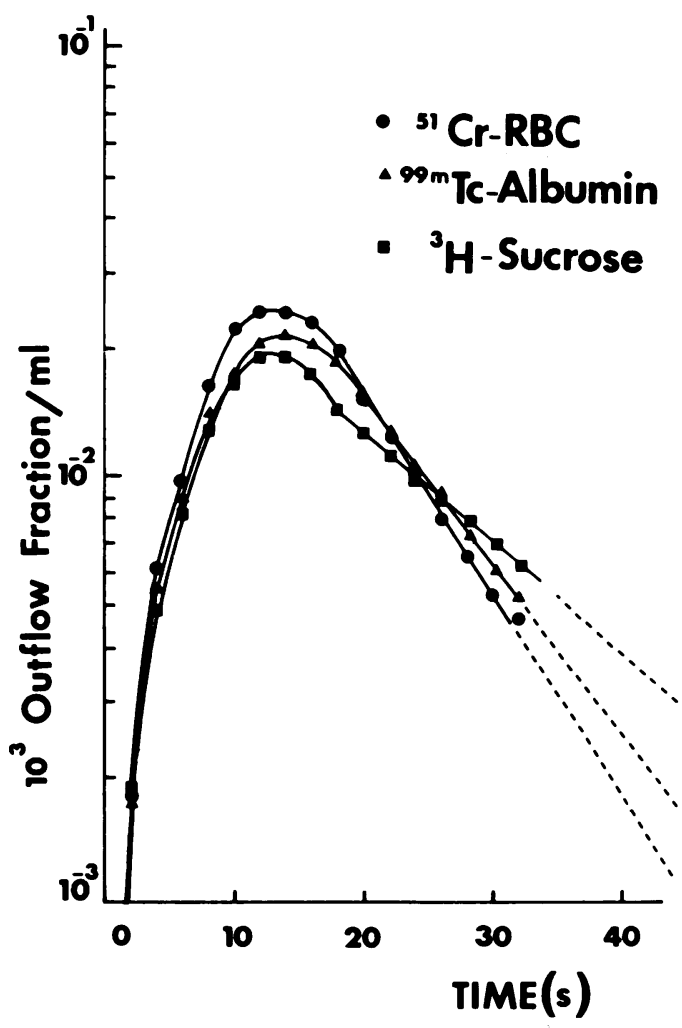

Patient no. 10

Figure 5 Labeled RBC, albumin, and sucrose outflow fraction per milliliter curves obtained in patient 10 . flow-limited distribution into its accessible extravascular space; the transformed labeled sucrose curves differed systematically from the labeled RBC curves and the flow-limited model could not be applied for labeled sucrose curve analysis. The changes in shape appeared to be related systematically to the changes in the $\gamma_{\mathrm{ALB}}$ found in these nine cirrhotic patients. The changes could be characterized by examining: $(a)$ the position in time of the peak of the sucrose curve $\left(t_{\mathrm{P} \text { SUC }}\right.$ [sucrose, SUC]) with respect to the peak of RBC curve $\left(t_{\mathrm{PRBC}}\right)$; and $(b)$ the relative magnitude of the peak sucrose concentration or the apparent $\left(1+\gamma_{P S U C}\right)$ (Table II). The upper panel of Fig. 6 shows the excess sucrose peak transit time ratio, $\left(t_{\mathrm{PSUC}}-t_{\mathrm{PRBC}}\right) / t_{\mathrm{PSUC}}$, as a function of $\gamma_{\mathrm{ALB}}$ values: at large $\gamma_{\mathrm{ALB}}$ value, a large delay in the time of the peak concentration of labeled sucrose is found with respect to that of labeled $\mathrm{RBC}$; and, as the $\gamma_{\text {ALB }}$ value becomes smaller, a progressive convergence of the time of their peak concentrations is observed. In the lower panel of Fig. 6, the apparent $\left(1+\gamma_{\mathrm{P} \text { SUC }}\right)$, as calculated by the ratio of the peak $\mathrm{RBC}$ concentration over the peak sucrose concentration, is plotted against $\gamma_{\text {ALB }}$. A bimodal relationship can be observed. At large $\gamma_{\text {ALB }}$ values, the peak ratio is intermediate, approaching values found in the normal dog liver (2.0 to 2.5) (Goresky, unpublished data), whereas, as $\gamma_{\text {ALB }}$ values become smaller, the peak ratio progressively increases to very large values until, at the lowest $\gamma_{\text {ALB }}$ values, the peak ratio abruptly decreases to very small values; the peak sucrose is now 

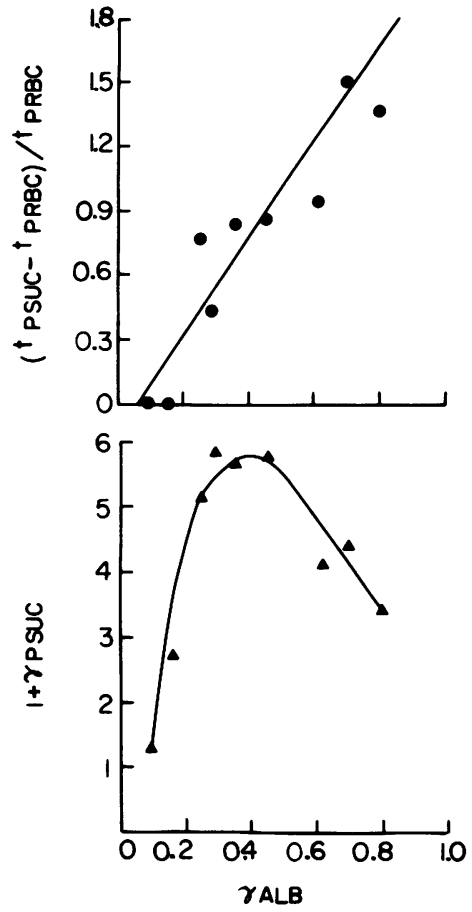

Figure 6 Upper panel: plot of the excess sucrose peak transit time ratio $\left(t_{\mathrm{PSUC}}-t_{\mathrm{P} \mathrm{RBC}}\right) / t_{\mathrm{PRBC}}$ vs. $\gamma_{\mathrm{ALB}}$. The correlation coefficient was $0.876(n=9, P<0.001)$. Lower panel: plot of the apparent peak ratio value for sucrose, $1+\gamma_{P S U C}$, vs. $\boldsymbol{\gamma}_{\text {ALB }}$.

ahead of that of labeled albumin and the downslope is biexponential (patients 10 and 17) (Fig. 5). This outflow profile, in this extreme case, is close to the one observed in the dog coronary circulation (10), a tight capillary circulation poorly permeable to sucrose. In this organ, the labeled sucrose outflow profile can be resolved in two parts: a throughput component, interpreted as labeled sucrose that has come through the coronary circulation without leaving the capillaries; and an exchanging component, interpreted as labeled sucrose that has penetrated an extravascular space and then emerges later on (10). The outflow profile obtained in these two patients may well have resulted from a similar phenomenon.

The recovery of labeled sucrose with respect to that of the labeled RBC was $0.94 \pm 0.15$ (values ranged from 0.75 to 1.18 ). In some of the protracted labeled sucrose curves, the appearance of recirculation on the downslope was discerned with difficulty, but extrapolation was nevertheless carried out in these cases.

\section{Studies characterized by bimodal outflow dilution curves}

The outflow curves for labeled RBC, albumin, and sucrose, observed in the two bimodal experiments, are illustrated in Fig. 7. Both patients had postnecrotic cirrhosis. In each case the labeled RBC, albumin, and sucrose dilution curves exhibit two parts: an earlier component, related in time to the peak of labeled RBC curve, and a later component, related to a bulge on the later downslope of the labeled RBC curve. The early peaks of the labeled albumin and sucrose curves, which are clearly related to the labeled RBC peak in time, appear to represent predominantly throughput material, coming through a well-capillarized less permeable part of the vascular bed. In the second or later components of the outflow profiles, the labeled albumin and labeled sucrose activities are delayed with respect to the labeled $\mathrm{RBC}$, with the delay in time and diminution in magnitude being larger for the labeled sucrose. The characteristics of the relations between the later parts of the curves in these experiments correspond to those expected to result from diffusion of albumin and sucrose labels during their propagation through a more normal liver sinusoidal bed. The curves in these cases thus exhibit clear evidence of heterogeneity; the groups of vascular transit times correspond to two different kinds of channels in these livers.

\section{Morphological findings in the cirrhotic liver}

Surgical biopsies, fixed for ultrastructural examination, were available in four patients, three with al-
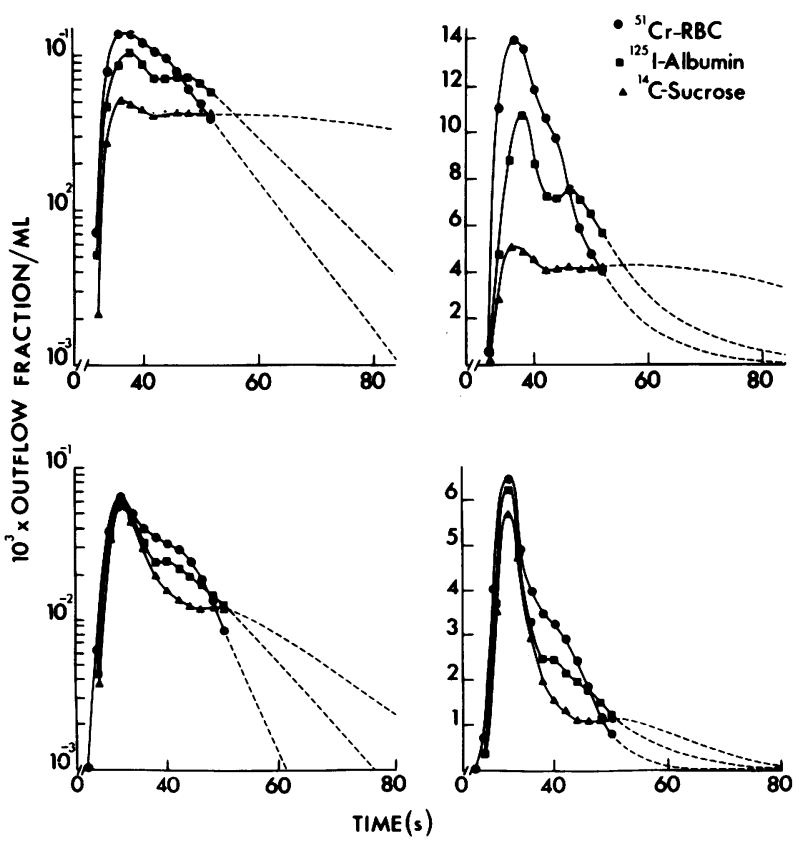

Figure 7 Bimodal labeled RBC, albumin, and sucrose outflow fraction per milliliter curves obtained in patient 24 (upper panels) and patient 25 (lower panels). 
coholic cirrhosis (patients 14,16, and 18), and one with postnecrotic cirrhosis (patient 24), and also in four additional patients with micronodular alcoholic cirrhosis. A set of representative morphological findings is displayed in Fig. 8.

In the biopsy specimens examined from patients with alcoholic cirrhosis, a stereotyped set of alterations was found in the microvasculature. Typically a micronodule exhibited a marginal regenerative area perfused with capillaries rather than sinusoids, and a central trabecular area in which sinusoids were present. The sinusoids were fenestrated in a normal fashion (Fig. 8A), and exhibited the kind of structure that provides the morphological base for flow-limited distribution of diffusible labels. The capillaries exhibited a continuous endothelial lining lacking fenestra, supported by a basement membrane and collagenous tissue (Fig. $8 \mathrm{~B}$ ). The continuous lining of the capillaries would be expected to form a permeability barrier and to be especially tight for labeled albumin and for protein-bound materials, such as ICG. The structure is such that, along a single vascular pathway, capillary and sinusoidal structures will be encountered in sequence. Variations in the degree of the capillary change would then be expected to produce a spectrum of $\gamma_{\mathrm{ALB}}$ and ICG extraction values and, in the highly capillarized extreme, to result in low $\gamma_{\mathrm{ALB}}$ values and low ICG extractions.

Biopsy material fixed for electron microscopy was also a vailable from patient 24 , one of the patients with postnecrotic cirrhosis and bimodal dilution curves. The specimen showed, at lower power, broad bands of scars interspersed with large areas of normal sinusoids, in which, at high power, no capillary changes were found.

\section{DISCUSSION}

In the normal liver, plasma-dissolved substances gain access to the extravascular space (the space of Disse) through fenestrae in and between the endothelial cells; therefore the cell membrane is the only barrier between the plasma content and the interior of the hepatocytes. In the normal dog liver, by using the multiple-indicator dilution technique, the behavior of substances such as labeled albumin and sucrose into the extravascular space has been shown to be compatible with a flow-limited system, without significant limitation of the diffusion into their respective spaces of distribution (5).

Using this technique, we have studied the hepatic microcirculation in a group of 25 cirrhotic patients. In 23 of these patients, unimodal outflow dilution curves were obtained, compatible in form with flow- limited distribution of labeled albumin into its space of distribution. However, there was a considerable variation in the space ratio values for albumin $\left(\gamma_{\mathrm{ALB}}\right)$, indicating that in some of the cirrhotic livers the space immediately accessible to labeled albumin has become progressively restricted. In a few of the cirrhotic patients, the relation between the outflow patterns for labeled RBC and albumin was similar to that observed in a tight capillary circulation such as heart and lung $(6,14)$. In these other organs, the lining endothelium is continuous and not perforated; it is impermeable to labeled albumin over the time of a single passage transit. Consequently, in these organs there is only a minimal delay in, and decrease in magnitude of, the labeled albumin curve with respect to that of labeled $\mathrm{RBC}$, due to intravascular separation (6). In the cirrhotic patients with the lowest $\gamma_{\text {ALB }}$ values, we would therefore expect that virtually all the labeled albumin remained in the vascular compartment.

The relation between data obtained from the labeled RBC and albumin curves and the ICG extraction and portohepatic gradient was explored. Considering first the portohepatic gradient used as an index of the severity of portal hypertension, there was a tendency for the deviation of the $\gamma_{\mathrm{ALB}}$ from the normal extreme to be small in patients with a low portohepatic gradient and large in those with a high portohepatic gradient, but there was a substantial scatter in the data. In contrast, there was a tight correlation between the decrease in $\gamma_{\mathrm{ALB}}$ and the decrease in extraction of ICG across the liver. ICG is bound to plasma proteins (15) and its extraction would be expected to be facilitated if the proteins carrying it were able to approach the sinusoidal surfaces of the liver cells (as is ordinarily the case). The reduced extraction of ICG may be accounted for by an impaired access of this proteinbound dye to the liver cell surface.

Data obtained from labeled sucrose studies in nine cirrhotic patients showed that the diffusion of sucrose, unlike that of albumin, was no longer compatible with a system where flow was the only limiting factor. It appeared that sucrose, a much smaller molecule than albumin, was still able to diffuse into an interstitial space not accessible to albumin, but that diffusion was then limited by a new "barrier" with restricted permeability. The change in shape of labeled sucrose curves could be related systemically to the changes in the space of distribution of albumin: With the decrease in the $\gamma_{\mathrm{ALB}}$ values, the peak of the labeled sucrose curve advanced in time and decreased in magnitude, and at very low $\gamma_{\mathrm{ALB}}$ values, the pattern of the labeled sucrose curve was compatible with a two-component profile, a large throughput component, and a small exchanging component. The progressive change in the shape of the sucrose curve corresponds to that pre- 

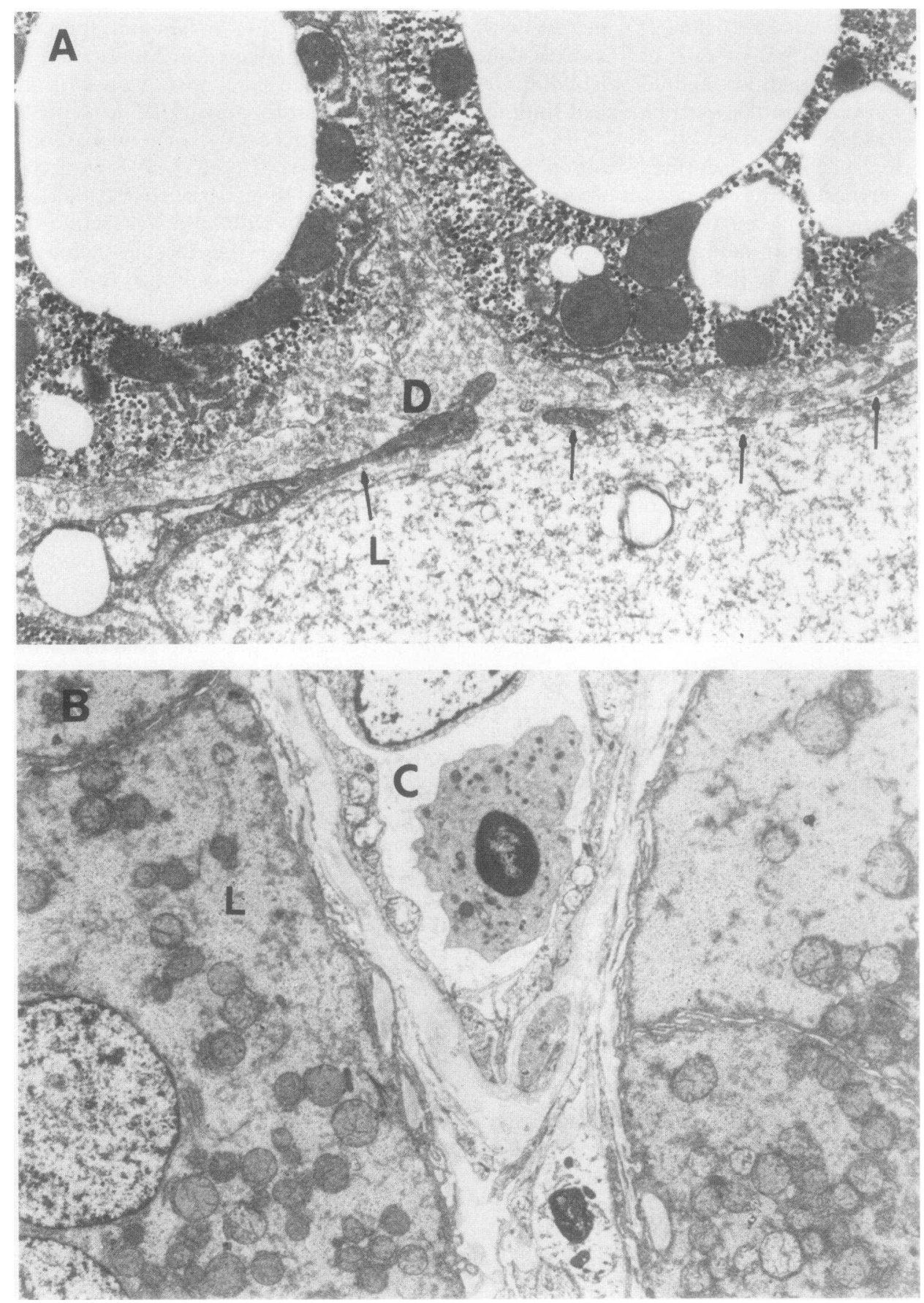

Figure 8 Representative electron microscopic pictures that show the microvascular changes associated with cirrhosis obtained from patient 16 . A. Electron micrograph $(\times 9,900)$ from the area of remaining trabecular liver, in which the sinusoidal sieve plate (arrows) is shown separating the space of Disse (D) from the sinusoidal lumen $(\mathrm{L})$. Triglyceride droplets are seen in the liver cells. B. A capillary is seen in this electron micrograph $(\times 4,900)$ between regenerative liver parenchymal cells. The capillary lumen $(\mathrm{C})$ is separated from the liver cells $(\mathrm{L})$ by the nonfenestrated endothelial cell, a basement membrane, and a layer of fibrillary collagen. The liver cells have a flattened surface membrane with reduced numbers of surface villi. 
dicted when the permeability of a limiting exchange barrier is progressively decreased (16). In the low $\gamma_{\mathrm{ALB}}$ extreme, the form of the outflow curves was compatible with a major limitation to the diffusive exchange of labeled sucrose across the walls of the microvasculature. At the same time, the potential contribution of intrahepatic shunting to the present findings is difficult to appraise. However, a positive correlation would be expected between the $\left(1+\gamma_{\mathrm{ALB}}\right)$ and $\left(1+\gamma_{P \text { SuC }}\right)$ values, if intrahepatic shunting was a major factor in the change in shape of both diffusible label curves. The relationship observed between $\left(1+\gamma_{\mathrm{ALB}}\right)$ and $\left(1+\gamma_{\mathrm{P} \text { suC }}\right)$ values, shown in Fig. 6 , is more suggestive of a progressive limitation of diffusion of labeled sucrose into its space of distribution rather than shunting.

The general hypothesis that then arises from the data is that as cirrhosis evolves, a change in the microcirculatory bed occurs such that a second "barrier" acts to restrict the access of albumin and protein-bound substances, such as ICG, to the interstitial space while limiting the diffusion of sucrose into that space. Exchange in the cirrhotic liver then comes to resemble that found in other tissues with a tight capillary system $(6,17)$.

The hypothesis of a functional two-barrier system is further strengthened by the ultrastructural alterations found in our patients and first described by Schaffner and Popper (3), that in long standing cirrhosis, collagenization of the interstitial space and formation of a basement membrane at the margin of the microvascular channels occur, with subsequent capillarization of the hepatic sinusoids $(3,18)$. These vascular changes tend to be regional, within the cirrhotic nodule $(19,21)$; in our biopsy studies we found that channels become capillarized in the pseudoacinar-regenerative areas at the periphery of the nodule and that the more normal sinusoidal sieve plates remained preserved in the center of the nodule. This kind of distribution provides a morphological base for the spectrum of changes observed. Capillarization presumably proceeds from the periphery to the center of the nodule as the cirrhotic process advances. Quantitative morphometric data will ultimately need to be secured to characterize this process. The task will be difficult, however, because a biopsy sample may not be representative of the entire liver, and transmission electron microscopic grids are so small that they often contain only a few microvascular channels.

In these studies the presence of intrahepatic large vessel shunting was not directly appraised by the simultaneous use of large labeled microspheres $(22,23)$. Their existence could be expected to further exacerbate the functional effects of capillarization. In a large group of patients with alcoholic liver diseases, portal vein injection of $25-\mu \mathrm{m}$ microspheres indicated that an average of $12 \%$ of the blood was shunted through large vessels (22). In another study involving five cirrhotic patients, Groszmann et al. (23) found shunt values ranging from 6 to $34 \%$ after injection of labeled microaggregates into the hepatic artery.

In our 25 studies, two sets of multiple-indicator dilution curves were bimodal, one in which a hepatic artery injection was used (patient 24) and the other in which a portal vein injection was used (patient 25). The anatomical basis for the bimodal curves was in each case macronodular postnecrotic cirrhosis (large bands of scars were present between the nodules in each case). We were able to analyze the bimodal curves (displayed in Fig. 7) only descriptively. In patient 24 , a smaller, and in patient 25 , a larger, proportion of the early simultaneous components of the RBC and diffusible (albumin and sucrose) tracers appearing at the outflow had a conformation that may well represent tracers shunted through large vessels. The exact proportion is difficult to quantitate, because the displacement of the diffusible tracer from the vascular reference is such that some proportion of the tracer must also have come through poorly permeable exchanging vessels. Shunt vessels were probably present in the broad bands of scars but were not demonstrated morphologically. In terms of our overall results, we would expect that the large shunting effect reported by others $(22,23)$ would be particularly likely to occur in those patients who exhibit bimodal dilution curves.

Small shunts would not be easily discernible using the multiple-indicator dilution technique without adding large labeled microspheres to the other tracers. In studies with the lowest $\gamma_{\mathrm{ALB}}$, large vessel shunting, if present, and capillary throughput components may be so intimately intermixed that unimodal labeled RBC and albumin curves result; the presence and extent of the former needs experimental appraisal. Further systematic studies that include large labeled microspheres will need to be carried out in cirrhotic patients. In the last patient included in the present study (No. 23), a second set of indicator dilution studies was obtained after injection of labeled RBC and ${ }^{99 \mathrm{~m}} \mathrm{Tc}$-labeled albumin microspheres $(20 \mu \mathrm{m}, 3 \mathrm{M}$ Co., $3 \mathrm{M}$ Center, St. Paul, MN) into the hepatic artery. In this patient, with unimodal RBC, albumin, and sucrose curves and an intermediate $\gamma_{\mathrm{ALB}}$, no ${ }^{99 \mathrm{~m}} \mathrm{Tc}$ activity was recovered in the hepatic venous blood during the passage of labeled RBC. The study effectively demonstrates the absence of intrahepatic shunts larger than $20 \mu \mathrm{m}$ in this patient and introduces the possibility that shunts may not be present in appreciable proportion in the unimodal group. 
Using the multiple-indicator dilution technique, we characterized events occurring in the microvascular bed of a group of cirrhotic patients and found major alterations in the liver microcirculation. The data are compatible with two kinds of alterations, capillarization of sinusoids with collagenization of sinusoids (that is, with imposition of a new barrier between vascular and interstitial spaces), and with intrahepatic shunts. The combination of these two kinds of alterations appears to account for the progressive limitation of exchange in the microvascular bed of the cirrhotic liver (24). From the data it appears that capillarization and collagenization of hepatic sinusoids are of particular importance in alcoholic liver cirrhosis, and that large intrahepatic shunts might be more frequent in postnecrotic liver cirrhosis.

\section{ACKNOWLEDGMENTS}

The authors thank Dr. André Viallet for his criticism in reviewing the manuscript. We thank Brita Nadeau, Antoine Brault, Robert Boileau, and François Carrière for their superb technical assistance and Margaret Mulherin and Françoise Trotier for typing this manuscript. Electron micrographs were prepared by Adriana Torrisi.

The financial support for these studies was provided by the Medical Research Council of Canada and the Québec Heart Foundation.

\section{REFERENCES}

1. Huet, P.-M., D. Marleau, P. Lavoie, and A. Viallet. 1976. Extraction of ${ }^{125}$ I-albumin microaggregates from portal blood: an index of functional portal blood supply in cirrhotics. Gastroenterology. 70: 74-81.

2. Branch, R. A., and D. G. Shand. 1976. Propranolol disposition in chronic liver disease: a physiological approach. Clin. Pharmacokinet. 1: 264-279.

3. Schaffner, F., and H. Popper. 1963. Capillarization of hepatic sinusoids. Gastroenterology. 44: 239-242.

4. Popper, H., H. Elias, and D. E. Petty. 1952. Vascular pattern of the cirrhotic liver. Am. J. Clin. Pathol. 22: 717-729.

5. Goresky, C. A. 1963. A linear method for determining liver sinusoidal and extravascular volumes. Am. J. Physiol. 204: 626-640.

6. Goresky, C. A., J. W. Warnica, J. H. Burgess, and B. E. Nadeau. 1975. Effect of exercise on dilution estimates of extravascular lung water and on the carbon monoxide diffusing capacity in normal adults. Circ. Res. 37: 379389.

7. Huet, P.-M., A. DuRéau, and D. Marleau. 1979. Arterial and portal blood flow supply in cirrhosis: a functional evaluation. Gut. 20: 792-796.

8. Meier, P., and K. L. Zierler. 1954. On the theory of the indicator-dilution method for measurement of flow and volume. J. Appl. Physiol. 6: 631-744.
9. Taylor, G. 1967. The dispersion of soluble matter in solvent flowing slowly through a tube. Proc. Roy. Soc. Ser. A. 219: 186-203.

10. Goresky, C. A., G. G. Bach, and B. E. Nadeau. 1973. On the uptake of materials by the intact liver. The concentrative transport of Rubidium 86. J. Clin. Invest. 52: 975-990.

11. Caesar, J., S. Shaldon, L. Chiandussi, L. Guevera, and S. Sherlock. 1961. The use of indocyanine green in the measurement of hepatic blood flow and as a test of hepatic function. Clin. Sci. (Lond.). 21: 43-57.

12. Brace, R. A. 1977. Fitting straight lines to experimental data. Am. J. Physiol. 233: R94-R99.

13. Benhamou, J.-P., F. Nicollo, A. Urbanczyk, and R. Fauvert. 1965. Le transit à travers le foie humain des érythrocytes et d'albumine: essai de dissection hémodynamique du foie. Rev. Fr. Etud. Clin. Biol. 10: 291-298.

14. Rose, C. P., C. A. Goresky, and G. G. Bach. 1977. The capillary and sarcolemmal barriers in the heart: an exploration of labeled water permeability. Circ. Res. 41: 515-533.

15. Baker, K. J. 1966. Binding of sulfobromophthalein (BSP) sodium and indocyanine green (ICG) by plasma $\alpha_{1}$ lipoprotein. Proc. Soc. Exp. Biol. Med. 122: 957-963.

16. Goresky, C. A., W. H. Ziegler, and G. G. Bach. 1970. Capillary exchange modeling. Barrier-limited and flowlimited distribution. Circ. Res. 27: 739-764.

17. Rose, C. P., C. A. Goresky, and G. G. Bach. 1977. Constraints on the uptake of labeled palmitate by the heart: the barriers at the capillary and sarcolemmal surfaces and the control of intracellular sequestration. Circ. Res. 41: 534-545.

18. Orrego, H., L. M. Blendis, I. R. Crossley, A. Medline, A. Macdonald, S. Ritchie, and Y. Israel. 1981. Correlation of intrahepatic pressure with collagen in the Disse space and hepatomegaly in humans and in the rat. Gastroenterology. 80: 546-556.

19. Phillips, M. J., and J. W. Steiner. 1965. Electron microscopy of liver cells in cirrhotic nodules. Am. J. Pathol. 46: 985-1005.

20. Steiner, J. W., A.-M. Jézéquel, M. J. Phillips, K. Miyai, and K. Arakawa. 1965. Some aspects of the ultrastructural pathology of the liver. In Progress in Liver Diseases. H. Popper and F. Schaffner, editors. Grune \& Stratton, Inc., New York. 2: 303-372.

21. Lapis, K. 1979. Cirrhosis. In Electron Microscopy in Human Medicine. The Liver, the Gallbladder, and the Biliary Ducts. J. V. Johannessen, editor. McGraw-Hill International Book Company, New York. 8: 158-189.

22. Hoefs, J., I. Sakimura, and T. Reynolds. 1978. Direct measurement of intrahepatic shunting by the portal vein injection of microspheres. Gastroenterology. 75: 968 (Abstr.)

23. Groszmann, R. J., D. Kravetz, and O. Parysow. 1977. Intrahepatic arteriovenous shunting in cirrhosis of the liver. Gastroenterology. 73: 201-204.

24. Wood, A. J. J., J-P. Villeneuve, R. A. Branch, L. W. Rogers, and D. G. Shand. 1979. Intact hepatocyte theory of impaired drug metabolism in experimental cirrhosis in the rat. Gastroenterology. 76: 1358-1362. 